\title{
Robust X-ray Angular Correlations for the Study of Meso-structures
}

\author{
Julien R. Lhermitte, ${ }^{a}$ Cheng Tian, ${ }^{a}$ Aaron Stein, ${ }^{a}$ Atikur Rahman, ${ }^{a}$ \\ Yugang Zhang, ${ }^{b}$ Lutz Wiegart,${ }^{b}$ Andrei Fluerasu, ${ }^{b}$ Oleg Gang ${ }^{a, c, d}$ And \\ Kevin G. YAGER ${ }^{a *}$ \\ ${ }^{a}$ Center for Functional Nanomaterials, Brookhaven National Laboratory, Upton, \\ New York 11973 US, ${ }^{b}$ National Synchrotron Light Source II, Brookhaven National \\ Laboratory, Upton, New York 11973 US, ${ }^{c}$ Department of Chemical Engineering, \\ Columbia University, New York, NY, 10027, USA US, and ${ }^{d}$ Department of Applied \\ Physics and Applied Mathematics, Columbia University, New York, NY, 10027 US. \\ E-mail: kyager@bnl.gov
}

\section{Simulations}

The mesoclusters were comprised of clusters of 3 spheres of radius 3 pixels arranged in an equilateral triangle of nearest neighbor separation 12 pixels, placed on a $2000 \times 2000$ pixel grid. The scattering cross-section is:

$$
I=\Phi_{0} \tan ^{2}\left(\frac{a}{R}\right) \frac{d \sigma}{d \Omega}
$$

where $\Phi_{0}$ is the flux (photons $/ \mathrm{s} / \mathrm{m}^{2}$, assumed constant over the samples measured), $a$ the size of a pixel $(75 \mu \mathrm{m}$ for EIGER $4 \mathrm{M})$ and $R$ is the sample to detector distance $(4.81 \mathrm{~m})$. The scattering cross section, in the far field, is given by the Born 
approximation (Als-Nielsen \& McMorrow, 2011):

$$
\begin{aligned}
\frac{d \sigma}{d \Omega} & =\left|r_{0} \mathrm{~F}(\vec{q})\right|^{2} \\
\mathrm{~F}(\vec{q}) & =\int_{V} \delta \rho^{\prime}(\vec{q}) e^{i \vec{q} \cdot \vec{r}} d \vec{r} \\
\delta \rho^{\prime}(\vec{r}) & =\rho_{s}^{\prime}(\vec{r})-\rho_{m}^{\prime}(\vec{r}) \\
\rho_{s, m}^{\prime} & =\frac{f_{s, m}-\left|f_{s, m}^{\prime}\right|}{f_{s, m}} \rho_{s, m}
\end{aligned}
$$

where $r_{0}$ is the classical Thomson radius, $\rho$ is the electron density and $\delta \rho$ is the effective electron density difference between the sample $(\mathrm{s})$ and medium $(\mathrm{m})$, which depends on the energy dependent dispersion correction $f^{\prime}$ (Als-Nielsen \& McMorrow, 2011). Absorption is ignored due to the thin nature of the samples. Note, the absolute value is used due to the possible ambiguity in sign definition of $f^{\prime}$ (usually negative). The absolute scattering is not of importance so these will be ignored. The scattering is essentially the modulus squared of the Fourier transform of the electron density. It was obtained by running a 2 dimensional discrete Fourier transform of the projection of the electron density grid and taking the modulus squared of this result. This result was used for our model. For the case of noisy simulations, Poisson noise was introduced by random sampling of values from a Poisson distribution, whose mean count rate is defined by the computed scattering. Note that at high count rates, the noisy simulations should converge to the model. The code was written in the Python programming language, using the numpy library for numerical computations (Oliphant, 2007).

\section{Signal to Noise versus Background}

The signal-to-noise for simulated measurements of varying background were computed as outlined in the previous section for the computation of noisy simulations. Before adding Poisson noise, the absolute intensity was rescaled so as to roughly match typical experimental data (on the order of fractions of a count to thousands per pixel). 100 
different realizations were computed for each parameter (background and meso-cluster number). We simulated the signal-to-noise versus mesocluster number in the presence of a background and found that the optimal mesocluster number was somewhere in between 1 cluster and a large number of clusters.

The correlation functions were computed in three steps as follows. First, the images were smoothed with a Gaussian kernel of $\sigma=8.3$ effective radii in real space (10 pixels on the simulated detector, in reciprocal space). This was found to be the optimal smoothing value. Next, the scattering from each simulation was regridded into a $1000 \times 200$ grid of intensities $\mathrm{I}(q, \phi)$. The angular correlations were calculated by a convolution in angle $\phi$ :

$$
C_{q, q}(\Delta \phi)=(I(q, \phi) \otimes I(q, \phi))(q, \Delta \phi)
$$

They were averaged among the 100 realizations per parameter.Finally, the signal-tonoise metric was computed as described in section 2.5 of the main article for the wave vector $q$ that corresponds to the first order peak of the difraction patterns. The resultant metric is shown in figure 1.

Various backgrounds were chosen, such that the cluster was not visible on its own but its powder ring was visible when many clusters were added to the simulation. Sample images of the scattering are shown for two backgrounds, differing by a factor of four, for 1, 10 and 20 mesoclusters in figure 2 .

Roughly, it was found that the peak in the signal-to-noise (figure 1) occurred for a mesocluster number where the scattering from them was just visible above the background by eye. Thus, it is sensible to choose a mesocluster number such that its resultant intensity variation is just above the background noise.

IUCr macros version 2.1.10: 2016/01/28 


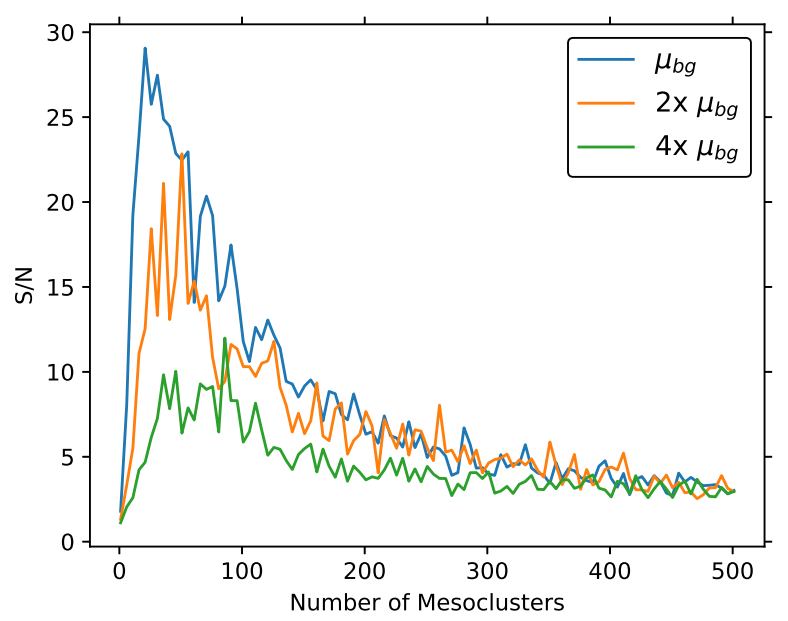

Fig. 1. A plot of the signal-to-noise versus cluster number with varying backgrounds. The backgrounds were chosen such that they affected the sample results.
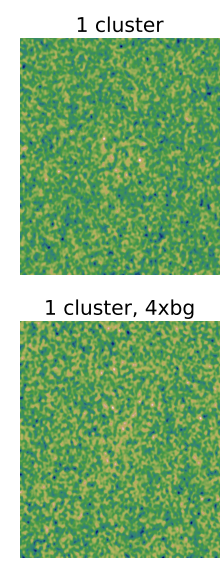

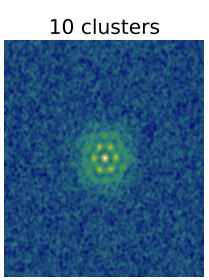

10 clusters, $4 \times \mathrm{xbg}$

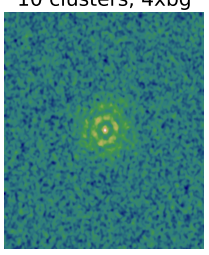

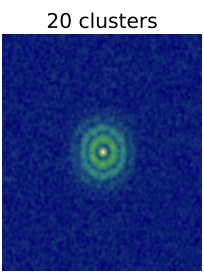

20 clusters, 4 xbg

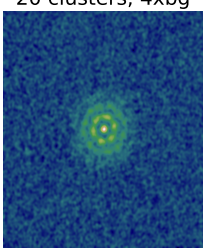

Fig. 2. A comparison of an instance of the signal-to-noise versus various backgrounds and cluster numbers. The clusters were randomly arranged in the array used for computation.

\section{Acknowledgements}

This research used resources of the Center for Functional Nanomaterials, and the National Synchrotron Light Source II, which are U.S. DOE Office of Science Facilities, operated at Brookhaven National Laboratory under Contract No. DE-SC0012704. 


\section{References}

Als-Nielsen, J. \& McMorrow, D. (2011). Elements of modern X-ray physics. John Wiley \& Sons.

Oliphant, T. E. (2007). Comput. Sci. Eng. 9(3), 10-20.

IUCr macros version 2.1.10: 2016/01/28 